\title{
Thermodynamic analysis of volatile organometallic fission products
}

\author{
John D. Auxier II ${ }^{1,2,3}$ (I) Jacob A. Jordan ${ }^{1,2} \cdot$ S. Adam Stratz ${ }^{1,2} \cdot$ Shayan Shahbazi $^{1,2}$ • \\ Daniel E. Hanson ${ }^{1,2} \cdot$ Derek Cressy $^{4} \cdot$ Howard L. Hall $^{1,2,3}$
}

Received: 29 May 2015/Published online: 17 December 2015

(c) The Author(s) 2015. This article is published with open access at Springerlink.com

\begin{abstract}
The ability to perform rapid separations in a post nuclear weapon detonation scenario is an important aspect of national security. In the past, separations of fission products have been performed using solvent extraction, precipitation, etc. The focus of this work is to explore the feasibility of using thermochromatography, a technique largely employed in superheavy element chemistry, to expedite the separation of fission products from fuel components. A series of fission product complexes were synthesized and the thermodynamic parameters were measured using TGA/DSC methods. Once measured, these parameters were used to predict their retention times using thermochromatography.
\end{abstract}

Keywords Nuclear forensics - Thermogravimetric analysis - Differential thermal analysis - Rapid separations

John D. Auxier II

jauxier@utk.edu

1 Department of Nuclear Engineering, University of Tennessee, 301 Middle Dr., Pasqua Nuclear Engineering Building, Knoxville, TN 37996, USA

2 Radiochemistry Center of Excellence (RCOE), University of Tennessee, 1508 Middle Dr., Ferris Hall, Knoxville, TN 37996, USA

3 Center for Public Policy, Institute for Nuclear Security, University of Tennessee, 1640 Cumberland Ave. Howard Baker Jr, Knoxville, TN 37996, USA

4 Department of Chemistry, University of Tennessee, 552 Circle Dr., Buehler Hall, Knoxville, TN 37996, USA

\section{Introduction}

The illicit use of nuclear material is one of the major challenges of the modern era. The threat of nuclear proliferation and nuclear terrorism is a continued and growing concern [1-5]. This threat has been recognized by Congress and was the primary motivation for the passage of the Nuclear Forensics and Attribution Act in 2010 [6]. This act called for the development of a credible capability for identifying sources of nuclear material used in an act of terrorism, and also acknowledged the challenge presented by the dwindling number of radiochemical programs and facilities in the United States. In an effort to improve the nuclear forensics capability, this work seeks to develop a method to reduce required to perform separation and detection of fission products found in a post-detonation scenario.

To address this need, this work will highlight the efforts to utilize thermodynamic measurements to allow the development of a technique known as thermo-chromatography $[2,3]$. Thermochromatography, or thermally driven physiochemical separation, is a technique that has been used almost exclusively in the superheavy element community due to its capability to utilize the volatile nature of carbonyl complexes giving rise to rapid separation times $[7,8]$. The focus of this work is to expand this application to new elements, in particular the rare earth elements, since they comprise the heavy end of the fission product curve. As such they can be utilized to identify a variety of nuclear forensic signatures based on their elemental presence and isotopic ratios.

In order to separate these fission products using thermochromatography, they must be volatile at a temperature that is attainable by the GCMS instrument. As oxides or chlorides, the fission product complexes simply non- 
volatile at the maximum operating temperature of a GCMS. To address this issue, a highly volatile ligand was attached to the fission products to allow for volatility at temperatures attainable by the GCMS instrument. A $\beta$ diketonate ligand was chosen for this process due to its high volatility and ease of synthesis.

The present work serves to approximate the thermodynamics, specifically the sublimation enthalpy, of various organometallic complexes comprised of rare earth metals with ligand. From this, the adsorption enthalpy can be empirically deduced. These thermodynamic quantities are essential in understanding the relative separations on a column. Such relationships can estimate retention times on a chromatography column. The goal of present and future work is to provide rapid separations of these common fission product compounds through experiment and simulation.

\section{Theory}

Thermochromatography is the separation of compounds via thermal mechanisms, either through thermal gradients or isothermal environments. Understanding the thermal characteristics of the compound is therefore necessary, with adsorption enthalpy being an important parameter when discussing column chromatography. Empirical correlations between the adsorption and sublimation enthalpy have been found for various heavy metal oxides by Eichler et al. [7] As part of this effort, the thermodynamic parameters, $\Delta G_{\text {sub }}, \Delta S_{\text {sub }}, \Delta H_{\text {sub }}$, or the Gibbs free energy of sublimation, entropy of sublimation, and enthalpy of sublimation, respectively, will be calculated using methods in thermogravimetric analysis (TGA). By understanding these values, the approximate retention times of the rare earth species can be predicted using kinetic models, or by thermodynamic methods such as those reported by Eichler et al. [7].

There is interest in the determination of rate-dependent parameters of non-isothermal sublimation by analysis of TG (thermogravimetric) curves and differential TG (DTG) curves. Most commonly found methods of analysis of such curves include the Horowitz-Metzger (HM), Coats-Redfern (CR) and Freeman-Carroll (FC) methods. Each uses a different approach in relating mass loss as a function of temperature change. Each equation can be graphed linearly, where the slope of the line can be used to solve for the sublimation enthalpy and the $y$-intercept used to find a pre-exponential factor, $Z$, used in the calculation of the sublimation entropy. The resulting equations are shown below, while their derivations are omitted for brevity [17-19].

\section{Horowitz-Metzger equation}

The Horowitz-Metzger equation is found via an integral method (Eq. 1):

$\ln \left[\frac{\left(1-\left(C_{\mathrm{HM}}\right)^{1-n}\right)}{(1-n)}\right]=\frac{E^{*} \theta}{\mathrm{RT}_{s}^{2}} ; \quad C_{\mathrm{HM}}=\frac{w-w_{\infty}}{w_{0}-w_{\infty}} ;$
$\quad \theta=T-T_{s}$

where $E^{*}$ is the activation energy and found from the slope, $n$ is the sublimation reaction order specific to the compound, $R$ is the ideal gas constant, and $T_{\mathrm{s}}$ is the sublimation temperature, or the temperature at the peak on the DTG curve. The weight of the sample is represented as $w$, while the weight at beginning and at completion are represented as $w_{0}$ and $w_{\infty}$, respectively. The pre-exponential factor, $Z$, is calculated using Eq. 2.

$Z=\frac{E^{*} \beta}{\mathrm{RT}_{s}^{2}} \exp \left(\frac{E^{*}}{\mathrm{RT}_{s}}\right)$

\section{Coats-Redfern equation}

The Coats-Redfern equation is found via an integral method using Eq. 3.

$$
\begin{gathered}
\log \left[\frac{\left(1-\left(1-C_{\mathrm{CR}}\right)^{1-n}\right)}{(1-n) T^{2}}\right]=\log \left[\frac{\mathrm{ZR}}{\beta E^{*}}\left(1-\frac{2 \mathrm{RT}_{s}}{E^{*}}\right)\right] \\
-\frac{E^{*}}{2.3 .3 \mathrm{RT}} ; \quad C_{\mathrm{CR}}=\frac{w_{0}-w}{w_{0}-w_{\infty}}
\end{gathered}
$$

where the variables are as above, $Z$ is the pre-exponential factor in $\mathrm{s}^{-1}$ and can be found from the $y$-intercept, and $\beta$ is the heating rate in ${ }^{\circ} \mathrm{C} / \mathrm{s}$.

\section{Freeman-Carroll equation}

The Freeman-Carroll equation is found via a differential method using Eq. 4.

$\ln \left[\frac{\mathrm{d} C_{\mathrm{FC}}}{\mathrm{d} T} /\left(1-C_{\mathrm{FC}}\right)^{n}\right]=\ln \left[\frac{z}{\beta}\right]-\frac{E^{*}}{\mathrm{RT}} ;$
$C_{\mathrm{FC}}=C_{\mathrm{CR}}=\frac{w_{0}-w}{w_{0}-w_{\infty}}$

where the variables are as above and the derivative of $C_{\mathrm{FC}}$ with respect to temperature is needed.

Regardless of method, the sublimation thermodynamic parameters can be found using Eqs. 5-7.

$\Delta H_{\text {sub }}=E^{*}-\mathrm{RT}_{s} \quad$ and $\quad \Delta S_{\text {sub }}=R \ln \left(\frac{h z}{k T_{s}}\right) \quad$ and

$\Delta G_{\text {sub }}=\Delta H_{\text {sub }}-T_{s} \Delta S_{\text {sub }}$

where the variables are as above, $h$ is Planck's constant, and $k$ is Boltzmann's constant. 

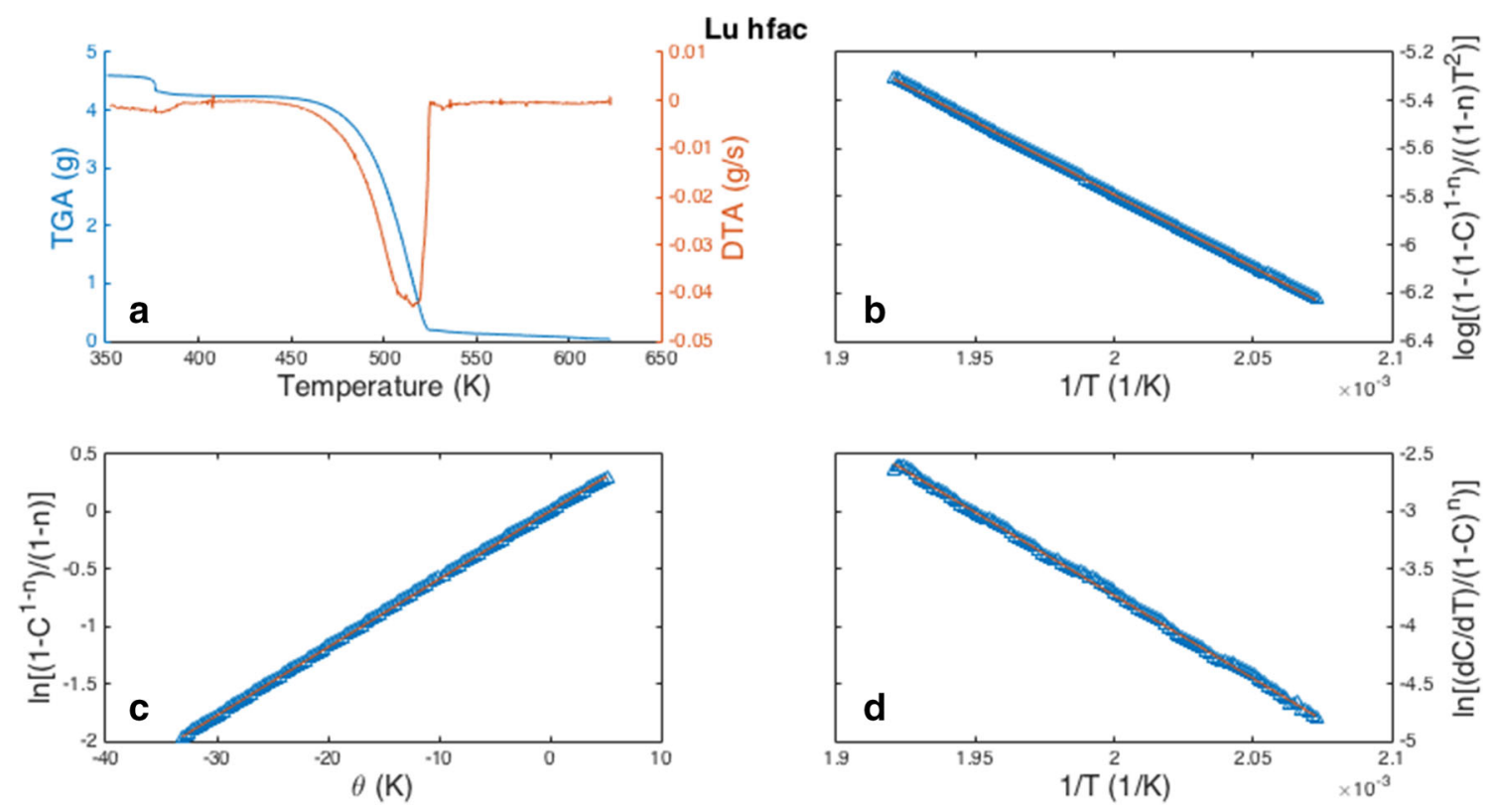

Fig. 1 a The TGA/DSC data for the $\mathrm{NH}_{4} \cdot \mathrm{Lu}[\mathrm{hfac}]_{4}$ compouds, b Coats-Redfern method, c Horowitz-Metzger method, and $\mathbf{d}$ Freeman Carroll method

\section{Experimental}

\section{Synthesis: hfac, hfod, and hdpm Complexes}

All reagents and solvents were used from commercial sources and used without further purification. The synthesis of the complexes followed that reported in the literature [9-16]. The rare earth oxides were dissovled in conc. $\mathrm{HCl}$ and were allowed to evaporate to produce $\mathrm{LnCl}_{3}$, where $\mathrm{Ln}$ represents any element in the rare earth series $\mathrm{La}-\mathrm{Lu}$, excluding $\mathrm{Ce}$ and $\mathrm{Pm}$. The 1,1,1,5,5,5-hexafluoroacetalacetone (hfac) was treated with $\mathrm{NH}_{4} \mathrm{OH}$ to form a white precipiate, as was the $6,6,7,7,8,8,8$-heptafluoro-2,2-dimethyl-3,5-octanedione (fod). The hfac or fod were then combined in a 4:1 molar ratio with the $\mathrm{LnCl}_{3}$ to produce the $\mathrm{Ln}[\mathrm{hfac}]_{4}$ or $\mathrm{Ln}[\mathrm{fod}]_{3}$. The 2,2,6,6-tetramethyl-3,5heptanedione (dpm) was combined under Ar with a $4 \mathrm{M}$ $\mathrm{NaOH}$ solution, which was stirred vigourously. The $\mathrm{LnCl}_{3}$ was added in 1:4 molar ratio as an aqueous solution to the reaction vessel and allowed to vacuum reflux overnight to prduce $\operatorname{Ln}[\mathrm{dpm}]_{3}$. The products were all collected via vacuum filtration.

\section{Thermodynamic measurements}

All TGA was performed on a Perkin Elmer Pyris 1 instrument. Samples of arbitrary mass between 3 and $6 \mathrm{mg}$ were run under nitrogen, held at $105{ }^{\circ} \mathrm{C}$ for $5 \mathrm{~min}$ or until signal equilibrated within $0.005{ }^{\circ} \mathrm{C}$, heated from 105 to $350{ }^{\circ} \mathrm{C}$ at $10{ }^{\circ} \mathrm{C} / \mathrm{min}$, then held at $350{ }^{\circ} \mathrm{C}$ for two additional minutes. This entails placing samples in high-temperature platinum (HT) pans with similar surface area, heating under desired temperature program, and repeating twice more for comparison.

Differential scanning calorimetry (DSC) is achieved via numerical differentiation of the raw TGA data curve with respect to temperature. A similar curve is utilized in the Freeman-Carroll method as described above. The analysis of the curves requires line-fitting, and is done so only over the region of sublimation, i.e., the entire peak of the DSC curve.

\section{Results and discussion}

\section{Thermodynamics results}

The TGA/DSC results are presented in the following figures. A number of methods for analyzing the TGA/DSC data have been reported in the literature, and for this work the methods reported by Freeman-Carroll, Horowitz-Metzger, and Coats-Redfern have been applied [17-19]. The results for $\mathrm{NH}_{4} \cdot \mathrm{Lu}[\mathrm{hfac}]_{4}$ have been shown as example (Fig. 1a-d), with all of the hfac compounds having a similar curve shape, and the results given in Table 1. The loss of $\mathrm{NH}_{4}^{+}$, or water, from the compounds is observed in the first region near $320 \mathrm{~K}$. The Horowitz-Metzger and 

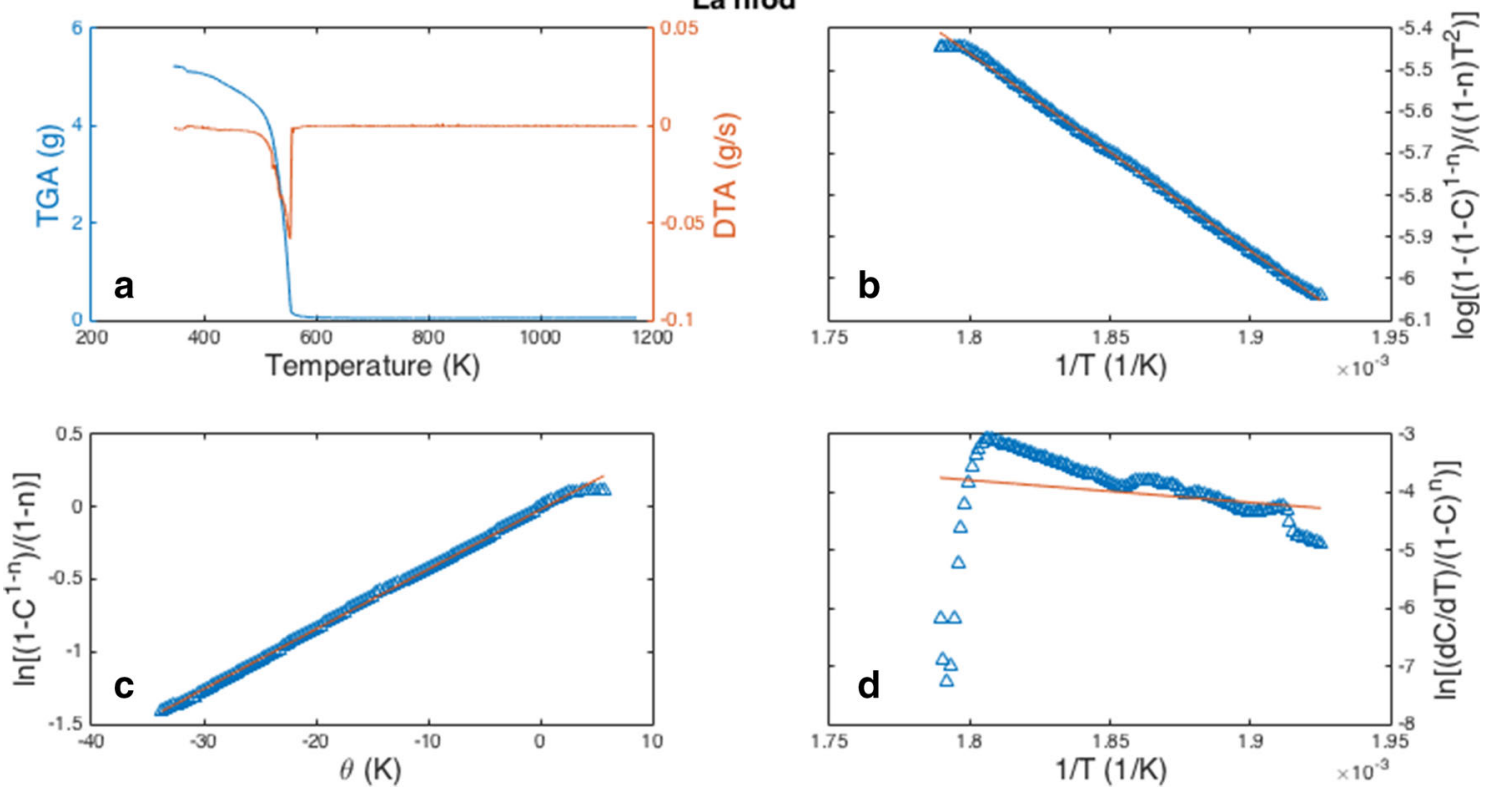

Fig. 2 a The TGA/DSC data for the $\mathrm{La}[\mathrm{fod}]_{3} \cdot \mathrm{H}_{2} \mathrm{O}$ compouds, b Coats-Redfern method, c Horowitz-Metzger method, d Freeman Carroll method

Coats-Redfern methods produce very similar plots and have similar results for their $\Delta G_{\text {sub }}, \Delta S_{\text {sub }}$, and $\Delta H_{\text {sub. The }}$ Freeman-Carroll method provides a very different treatment of the data and has different results. The large difference in mass between 480 and $520 \mathrm{~K}$ is the region where sublimation of the compounds occurs.

In a similar fashion, the $\mathrm{Ln}$ [fod] compounds were also analyzed via TGA/DSC, with $\mathrm{La}[\text { fod }]_{3} \cdot \mathrm{H}_{2} \mathrm{O}$ shown as example (Fig. 2a-d). Like the previous analysis, the Horowitz-Metzger method and the Coats-Redfern methods remain similar in their treatment of the data. The FreemanCarroll method, however does not fit the data nearly as well. The region between 380 and $400 \mathrm{~K}$ is likely where any additional water is removed from the complex, and the region between 550 and $600 \mathrm{~K}$ is where the fod compounds generally sublime.

The final compound analyzed was the $\mathrm{Ln}[\mathrm{dpm}]$ series of complexes, where the $\mathrm{Ho}[\mathrm{dpm}]_{3} \cdot \mathrm{H}_{2} \mathrm{O}$ is depicted in Fig. 3 as example. Like the hfac data, the dpm samples were very similar in the curve shape of the data across the series, and the full results are compiled in Table 1. Yet similar to the hfod samples, in the region between 380 and $400 \mathrm{~K}$ most of the water is removed, and the region between 480 and $520 \mathrm{~K}$ is the point at which the compounds sublime. Similarly, the Horowitz-Metzger and Coats-Redfern methods provide similar treatments to the data and similar results, while the Freeman-Carroll method gives a very different result as shown in Table 1.

The complete results from all of the TGA/DSC methods are presented in Table 1. The ionic radius of the method ion is given in angstroms, the $T_{s}$ is the temperature of sublimation, the range is the region of change (in $\mathrm{K}$ ), the $\Delta E$ is the energy of activation for the process (in J), the A or $\mathrm{Z}$ factor is a correction factor corresponds the method used to analyze the compound. HM corresponds to the Horowitz-Metzger, CR corresponds to the Coats-Redfern method, and FC corresponds to the Freeman-Carroll method.

The results of the table are shown graphically in Fig. 4, where the average (of the HM and CR methods) Gibbs' free energy of sublimation (abscissa) is plotted as a function of the ionic radius of the metal compounds.

The $\mathrm{Ln}[\mathrm{hfac}]$ compounds trend towards lower values of $\Delta G$, while the $\operatorname{Ln}[$ fod] compounds trend towards larger values as the ionic radius increases. The $\operatorname{Ln}[\mathrm{dpm}]$ compounds do not have an apparent trend as a function of atomic radius.

\section{Prediction of retention times}

The thermodynamic data can be used to predict the retention times in thermochromatographic experiments as noted by Eichler et al. [7]. The retention times can be determined using Eq. 8.

$$
\begin{aligned}
t_{r}= & \frac{L T_{0} \phi}{\bar{V}_{0} T_{\text {iso }}} \\
& \times\left(1+\frac{s}{v} \times \frac{V}{100 A} \times \exp \left(-\frac{\Delta H_{\text {ads }}^{0}}{R T_{\text {iso }}}\right) \times \exp \left(\frac{\Delta S_{\text {ads }}^{0}}{R}\right)\right)
\end{aligned}
$$



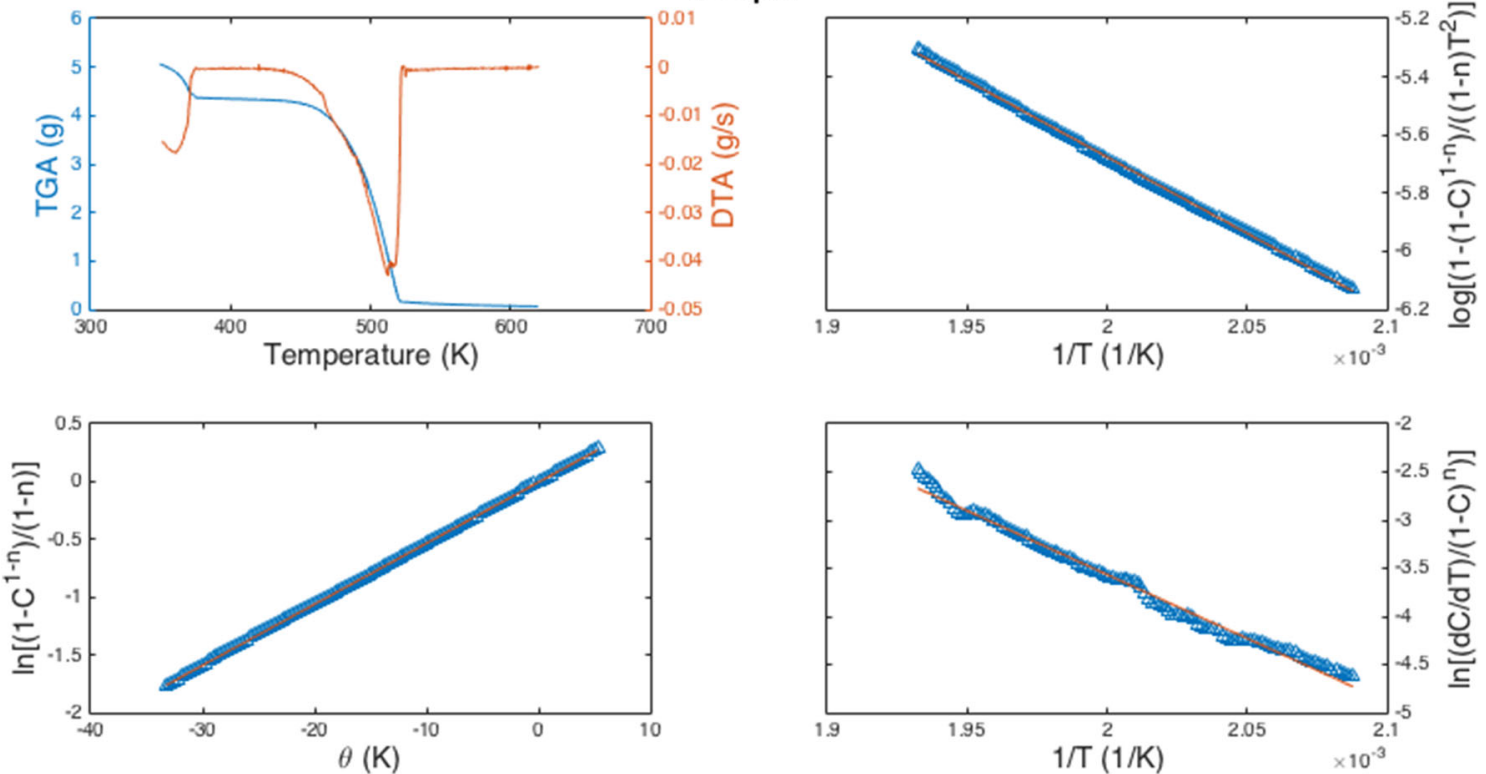

Fig. 3 a The TGA/DSC data for the $\mathrm{Ho}[\mathrm{dpm}]_{3} \cdot \mathrm{H}_{2} \mathrm{O}$ compouds, b Coats-Redfern method, c Horowitz-Metzger method, d Freeman Carroll method

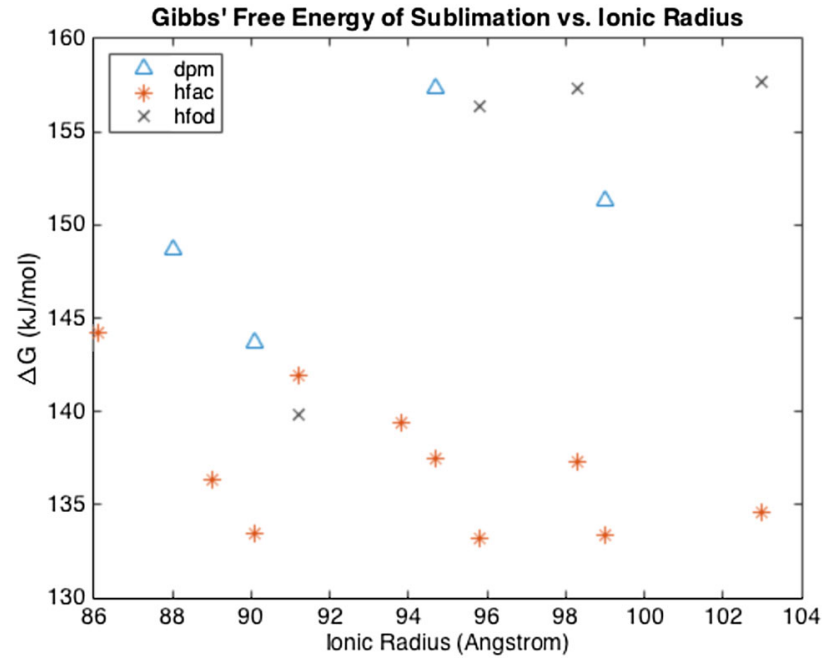

Fig. 4 The average (of the HM and CR methods) Gibbs' free energy of sublimation (ordinate) is plotted as a function of the ionic radius (abscissa)

where $L$ is the length of the column, $T_{0}$ is standard temperature $298.15 \mathrm{~K}, \phi$ is the free open cross-sectional area of the column, $V_{0}$-bar is the carrier gas flow at STP (standard temperature and pressure), $T_{\text {iso }}$ is the isothermal column temperature, $s$ is the open surface of column per $1 \mathrm{~m}$ column length, $v$ is the open volume of the column per $1 \mathrm{~m}$ column length, $V$ is the inner volume of the column, $A$ is the inner surface per $1 \mathrm{~m}$ of column length, and $R$ is the ideal gas constant. The entropy of adsorption can be calculated from the previous equation, while the enthalpy of adsorption can be found using Eq. 9.

$-\Delta H_{\text {ads }}^{0}=(2.9 \pm 16)+(.73 \pm .1) \times \Delta H_{\text {subl }}^{0}$

where the enthalpy of sublimation was taken from the thermodynamic models mentioned previously. The CoatsRedfern and the Horowitz-Metzger methods were used for calculation of the parameter, while the Freeman-Carroll was not used due to the inconsistent nature of parameter values obtained from the method. The calculation of $\Delta S_{\text {ads }}^{0}$ can be done using Eq. 10.

$\Delta S_{\mathrm{ads}}^{0}=R\left(\ln \left(\frac{100 A}{V \times v_{b}}\right) \times \sqrt{\frac{R \times T}{2 \times \pi \times M_{\mathrm{a}}}+\frac{1}{2}}\right)$

where the entropy of adsorption is related to $R$, the ideal gas constant, the area of the column, $A$, the volume of the column, $V$, the phonon frequency of the column material (e.g., quartz, etc.), $v_{b}$, the temperature, $T$, and the mass of the adsorbing material, $M_{\mathrm{a}}$. The approximate retention times using a thermochromatography unit fitted with a $30 \mathrm{~m} \mathrm{SiO}_{2}$ column operating at $150{ }^{\circ} \mathrm{C}$, with a flow rate of $0.8 \mathrm{~cm} / \mathrm{s}$ and an inner diameter of $0.5 \mathrm{~mm}$, were approximated and tabulated in Table 2. 
Table 1 The complete thermodynamic parameters for the $\operatorname{Ln}[\mathrm{hfac}], \operatorname{Ln}[$ fod], and Ln[dpm] compounds

\begin{tabular}{|c|c|c|c|c|c|c|}
\hline Comp. & Meth. & $T_{\mathrm{s}}(\mathrm{K})$ & Range (K) & $\Delta S_{\text {sub }}(\mathrm{kJ} / \mathrm{mol} \mathrm{K})$ & $\Delta H_{\text {sub }}(\mathrm{kJ} / \mathrm{mol})$ & $\Delta G_{\text {sub }}(\mathrm{kJ} / \mathrm{mol})$ \\
\hline Dy hfac & $\mathrm{CR}$ & 504 & $471-509$ & -0.1109 & 85.94 & 141.85 \\
\hline Dy hfac & HM & & & -0.0827 & 99.89 & 141.58 \\
\hline Dy hfac & $\mathrm{FC}$ & & & -0.1236 & 79.99 & 142.31 \\
\hline Dy hfod & $\mathrm{CR}$ & 500 & $466-505$ & -0.0796 & 100.23 & 140.01 \\
\hline Dy hfod & $\mathrm{HM}$ & & & -0.0491 & 115.24 & 139.78 \\
\hline Dy hfod & $\mathrm{FC}$ & & & -0.0561 & 111.71 & 139.75 \\
\hline Er hfac & $\mathrm{CR}$ & 484 & $451-489$ & -0.1445 & 66.76 & 136.75 \\
\hline Er hfac & $\mathrm{HM}$ & & & -0.1173 & 79.64 & 136.44 \\
\hline Er hfac & $\mathrm{FC}$ & & & -0.0624 & 105.53 & 135.75 \\
\hline Eu dpm & $\mathrm{CR}$ & 557 & $524-562$ & -0.1059 & 98.46 & 157.46 \\
\hline Eu dpm & $\mathrm{HM}$ & & & -0.0787 & 113.42 & 157.25 \\
\hline Eu dpm & $\mathrm{FC}$ & & & -0.0961 & 103.85 & 157.37 \\
\hline Eu hfac & $\mathrm{CR}$ & 488 & $455-493$ & -0.1533 & 63.34 & 138.18 \\
\hline Eu hfac & $\mathrm{HM}$ & & & -0.1264 & 76.07 & 137.76 \\
\hline Eu hfac & $\mathrm{FC}$ & & & -0.0077 & 132.84 & 136.62 \\
\hline Gd hfac & $\mathrm{CR}$ & 488 & $455-493$ & -0.2243 & 30.80 & 140.24 \\
\hline Gd hfac & $\mathrm{HM}$ & & & -0.2019 & 41.53 & 140.04 \\
\hline Gd hfac & $\mathrm{FC}$ & & & 0.0019 & 138.89 & 137.96 \\
\hline Ho dpm & $\mathrm{CR}$ & 512 & $479-517$ & -0.0928 & 96.38 & 143.92 \\
\hline Ho dpm & HM & & & -0.0637 & 111.04 & 143.65 \\
\hline Ho dpm & $\mathrm{FC}$ & & & -0.0735 & 105.96 & 143.62 \\
\hline Ho hfac & $\mathrm{CR}$ & 480 & $449-485$ & -0.0503 & 109.46 & 133.59 \\
\hline Ho hfac & HM & & & -0.0191 & 124.24 & 133.41 \\
\hline Ho hfac & $\mathrm{FC}$ & & & -0.0373 & 115.51 & 133.40 \\
\hline La hfac & $\mathrm{CR}$ & 474 & $442-479$ & -0.2058 & 37.91 & 135.45 \\
\hline La hfac & $\mathrm{HM}$ & & & -0.1818 & 48.93 & 135.12 \\
\hline La hfac & $\mathrm{FC}$ & & & -0.0309 & 118.58 & 133.20 \\
\hline La hfod & $\mathrm{CR}$ & 553 & $519-559$ & -0.1280 & 86.11 & 156.93 \\
\hline La hfod & HM & & & -0.1015 & 100.48 & 156.63 \\
\hline La hfod & $\mathrm{FC}$ & & & -0.2388 & 27.43 & 159.53 \\
\hline Lu hfac & $\mathrm{CR}$ & 516 & $482-521$ & -0.0633 & 111.67 & 144.33 \\
\hline Lu hfac & $\mathrm{HM}$ & & & -0.0327 & 127.24 & 144.11 \\
\hline Lu hfac & $\mathrm{FC}$ & & & -0.0546 & 116.10 & 144.25 \\
\hline Nd hfac & $\mathrm{CR}$ & 477 & $445-483$ & -0.2512 & 18.12 & 138.06 \\
\hline $\mathrm{Nd}$ hfac & $\mathrm{HM}$ & & & -0.2311 & 27.86 & 138.18 \\
\hline Nd hfac & $\mathrm{FC}$ & & & 0.0025 & 136.91 & 135.72 \\
\hline Nd hfod & $\mathrm{CR}$ & 560 & $527-565$ & -0.0743 & 116.49 & 158.11 \\
\hline Nd hfod & HM & & & -0.0443 & 132.74 & 157.56 \\
\hline Nd hfod & $\mathrm{FC}$ & & & 0.0674 & 194.22 & 156.45 \\
\hline Pr dpm & $\mathrm{CR}$ & 539 & $505-544$ & -0.0610 & 118.23 & 151.10 \\
\hline Pr dpm & $\mathrm{HM}$ & & & -0.0310 & 134.31 & 151.04 \\
\hline Pr dpm & $\mathrm{FC}$ & & & -0.1019 & 96.80 & 151.75 \\
\hline Pr hfac & $\mathrm{CR}$ & 477 & $445-482$ & -0.1344 & 70.55 & 134.59 \\
\hline Pr hfac & $\mathrm{HM}$ & & & -0.1050 & 83.82 & 133.86 \\
\hline Pr hfac & $\mathrm{FC}$ & & & 0.1323 & 194.71 & 131.66 \\
\hline Sm hfac & $\mathrm{CR}$ & 465 & $434-470$ & -0.2451 & 20.10 & 134.06 \\
\hline Sm hfac & $\mathrm{HM}$ & & & -0.2244 & 29.71 & 134.05 \\
\hline Sm hfac & $\mathrm{FC}$ & & & 0.0486 & 154.07 & 131.47 \\
\hline Sm hfod & $\mathrm{CR}$ & 551 & $518-556$ & -0.1348 & 82.23 & 156.53 \\
\hline Sm hfod & HM & & & -0.1084 & 96.46 & 156.20 \\
\hline Sm hfod & $\mathrm{FC}$ & & & -0.0892 & 107.21 & 156.35 \\
\hline Tm dpm & $\mathrm{CR}$ & 524 & $491-529$ & -0.1527 & 68.80 & 148.88 \\
\hline Tm dpm & $\mathrm{HM}$ & & & -0.1271 & 81.97 & 148.64 \\
\hline Tm dpm & $\mathrm{FC}$ & & & -0.1145 & 88.40 & 148.45 \\
\hline
\end{tabular}


Table 2 The calculated retention times for selected $\mathrm{Ln}[\mathrm{hfac}]$, Ln[fod], and Ln[dpm] compounds

\begin{tabular}{llll}
\hline & Ho[dpm] & La[fod] & Lu[hfac] \\
\hline MW $(\mathrm{g} / \mathrm{mol})$ & 734.37 & 1045.47 & 1025.24 \\
$\Delta H_{\text {subl }}(\mathrm{kJ} / \mathrm{mol})$ & 103.7 & 93.3 & 119.5 \\
$\Delta H_{\text {ads }}(\mathrm{kJ} / \mathrm{mol})$ & -78.6 & -71.0 & -90.1 \\
$\Delta S_{\text {ads }}(\mathrm{kJ} / \mathrm{mol} \mathrm{K})$ & -0.142 & -0.119 & -0.120 \\
$t_{\mathrm{r}}(\mathrm{s})$ & $1.496 \mathrm{E}+05$ & $2.965 \mathrm{E}+05$ & $5.789 \mathrm{E}+07$ \\
\hline
\end{tabular}

\section{Conclusions}

This work represents the first reports of the thermodynamic parameters of the $\beta$-diketonate complexes of $\mathrm{Ln}[\mathrm{hfac}$, Ln[hfod], and Ln[hdpm]. The thermodynamics have been used to begin to establish the feasibility of using thermochromatography as a technique to perform rapid separation of fission products. Knowledge of the thermodynamics of these compounds can be beneficial in developing approximations of retention times. Initial approximations from the model are much longer than hypothesized, and in initial chromatographic experiments, the times observed are much shorter. The observed discrepancy is largely due to the approximations for a variety of the theoretical parameters of the model and the differences in chemistry between the lanthanide $\beta$-diketonates and the super heavy elements. Future work will involve the refinement of the model's parameters and its operation, as well as the introduction and detection of the aforementioned compounds on an isothermal chromatographic column.

Acknowledgments This work was performed under grant number DE-NA0001983 from the Stewardship Science Academic Alliances (SSAA) Program of the National Nuclear Security Administration (NNSA). The views expressed are those of the authors and do not necessarily reflect those of the DOE or NNSA.

Open Access This article is distributed under the terms of the Creative Commons Attribution 4.0 International License (http://crea tivecommons.org/licenses/by/4.0/), which permits unrestricted use, distribution, and reproduction in any medium, provided you give appropriate credit to the original author(s) and the source, provide a link to the Creative Commons license, and indicate if changes were made.

\section{References}

1. Nuclear forensics: role state of the art, and program needs. http:// www.aaas.org/report/nuclear-forensics-role-state-art-programneeds. Accessed 4 April 2015

2. Garrison J, Hanson D, Hall H (2012) Monte Carlo analysis of thermochromatography as a fast separation method for nuclear forensics. J Radioanal Nucl Chem 291:885-894

3. Hanson D, Garrison J, Hall H (2011) Assessing thermochromatography as a separation method for nuclear forensics: current capability vis-à-vis forensic requirement. J Radioanal Nucl Chem 289:213-223

4. Schädel M, Shaughnessy DA (2011) The chemistry of superheavy elements. Springer, Heidelberg

5. Zvara I (2008) The inorganic radiochemistry of heavy elements. Springer, Dubna

6. Nuclear forensics and attributions act. https://www.govtrack.us/ congress/bills/111/hr730. Accessed 4 April 2015

7. Evan J, Yakushev A et al (2014) Synthesis and detection of a seaborgium carbonyl complex. Science 345:1491-1494

8. Yakushev A, Gates J et al (2014) Superheavy element flerovium (Element 114) is a volatile metal. Inorg Chem 53:1624-1629

9. Berg E, Acosta J (1968) Fractional sublimation of the B-diketone chelates of the lanthanide and related elements. Anal Chim Acta 40:101-113

10. Halverson F, Brinen J, Leto J (1964) Luminescence of europium hexafluoroacetylacetonate. J Chem Phys 40:2790-2792

11. Halverson F, Brinen J, Leto J (1964) Photoluminescence of lanthanide complexes. II. Enhancement by an insulating sheath. J Chem Phys 41:157-163

12. Kaz'mina N, Kupriyanova G, Troyanov S (2000) Crystal structure and vacuum sublimation of the product of reaction of yttrium hexafluoroacetylacetonate and copper acetylacetonate $\left[\mathrm{Y}(\mathrm{Hfa})_{3}\right.$ $\left.\left(\mathrm{H}_{2} \mathrm{O}\right) 2 \mathrm{Cu}(\mathrm{Acac})_{2}\right]$. Russ J Coord Chem 26:390-395

13. Ahmed Z, Iftikhar K (2010) Solution studies of lanthanide (III) complexes based on 1,1,1,5,5,5-hexafluoro-2,4-pentanedione and 1,10-phenanthroline part-I: synthesis, $1 \mathrm{H}$ NMR, $4 \mathrm{f}-4 \mathrm{f}$ absorption and photoluminescence. Inorg Chim Acta 363:2606-2615

14. Ahmed Z, Iftikhar K (2012) Synthesis, luminescence and NMR studies of lanthanide (III) complexes with hexafluoroacetylacetone and phenanthroline. Part II. Inorg Chim Acta. 392:165-176

15. Springer C, Meek D, Sievers R (1966) Rare earth chelates of 1,1,1,2,2,3,3-heptafluoro-7,7-dimethyl-,6-octanedione. Inorg Chem 6: $1105-1110$

16. Eisontraut K, Sievers R (1965) Volatile rare earth chelates. J Am Chem Soc 87:20-22

17. Horowitz H, Metzger G (1963) A new analysis of thermogravimetric traces. Anal Chem 35:1464-1468

18. Coats A, Redfern J (1965) Kinetic parameters from thermogravimetric data. Polym Lett 3:917-920

19. Freeman E, Carroll B (1969) Interpretations of the kinetics of thermogravimetric analysis. J Phys Chem 73:5-6 